\title{
A New Method of Determining Residual Thiosulfate in Processed Photographic Film
}

\author{
C. I. Pope ${ }^{1}$ \\ Institute for Basic Standards, National Bureau of Standards, Washington, D.C. 20234
}

(October 7, 1970)

\begin{abstract}
Chromatographic paper, saturated with ammonia, is used to extract the residual thiosulfate from the gelatin layer of processed film. The paper is treated in a silver nitrate solution and fixed in an ammonia-sodium chloride solution. Thiosulfate, if present, forms silver sulfide and the thiosulfate is determined by measuring the transmission density of the paper darkened by silver sulfide. The paper extraction method is simple, rapid, requires only a densitometer as special equipment, and is very sensitive especially when the transmission density is measured for two layers of the paper. No filters are required. The test readily reveals the uneven distribution of residual thiosulfate on the film. By selective removal of thiosulfate from the image silver by ammonia and potassium bromide solutions, it was shown that a small amount of thiosulfate was absorbed on the image silver immediately after processing.
\end{abstract}

Key words: Archival film; hypo; microfilm; permanent record film.

\section{Introduction}

Residual thiosulfate is readily determined in processed photographic papers by measuring the transmission density of the silver sulfide formed in situ by the reaction of the silver ion with the residual thiosulfate [1]. ${ }^{2}$ However, the transmission density of the silver sulfide in the gelatin layer of film is much lower for corresponding thiosulfate concentrations, making it difficult to use the density of the silver sulfide as a measure of residual thiosulfate concentration in film $[2,3]$.

It has been found that chromatographic paper wetted with an ammonia solution will absorb the residual thiosulfate from the gelatin layer of the film. Such paper was treated in silver nitrate solution forming silver sulfide in situ and the excess silver ion was removed by treating the paper in an ammonia solution. The transmission density of the silver sulfide was large enough to determine thiosulfate concentrations as low as $0.15 \mu \mathrm{g} / \mathrm{cm}^{2}$. The film was not destroyed in the test.

\section{Experimental Procedure}

Whatman No. 3 MM chromatographic paper $^{3}$ was selected for this study. The paper, which was $0.03 \mathrm{~cm}$ thick, withstood the treatments in the solutions and drying without distortion. A 2-percent ammonia solution was found to be very efficient in extracting the residual thiosulfate from the gelatin. The ammonia solution should either be used in a hood or covered during the test.

A strip of film 10 to $12 \mathrm{~cm}$ long and 1.6 to $2 \mathrm{~cm}$ wide was taped, emulsion side up, at the two ends to blotter

1 Retired. Present address: 2911 Dawson Ave., Silver Spring, Md. 20902.

2 Figures in brackets indicate the literature references at the end of this paper.

3 Commercial materials and equipment are identified in this paper in order to specify the experimental procedure adequately. Such identification does not imply recommendation nor endorsement by the National Bureau of Standards. paper resting on a level glass plate. The chromatographic paper, cut the width of the film sample and of a length to fit between the tape strips at the ends of the film, was soaked $3 \mathrm{~min}$ in about $100 \mathrm{ml}$ of a 2 -percent ammonia solution. Then the paper was grasped with tweezers at one end, drained briefly, and the other end brought in contact with the film, allowing the paper sample to roll down onto the film to prevent air bells. The paper was rubbed once gently with the back of the tweezers. The blotter paper absorbed any excess ammonia solution at the edges of the film. After $5 \mathrm{~min}$ the paper was removed and bathed $4 \mathrm{~min}$ in $100 \mathrm{ml}$ of a solution containing $10 \mathrm{~g}$ of silver nitrate and $30 \mathrm{ml}$ of glacial acetic acid per liter. A control strip was made by treating a sample of the chromatographic paper in the 2-percent ammonia solution $3 \mathrm{~min}$ before the test sample was removed from the film and the two paper samples were processed together. Next, the papers were placed in crystallizing dishes and treated 3 min in each of two solutions containing about $100 \mathrm{ml}$ of 5-percent ammonia solution and $1 \mathrm{~g}$ of sodium chloride per liter, to remove the excess silver ion. The solutions were stirred during treatments. Then the papers were removed from the ammonia solution with tweezers and placed on blotting paper but not blotted. The paper was turned over three or four times during a 10-min period and allowed to dry in the room atmosphere. The transmission density was measured for the control and test papers. The effective optical density of the silver sulfide was determined by subtracting the density of the control paper from that of the test paper. The corresponding residual thiosulfate concentration of the film was determined from a calibration curve.

It was found that 3 to 5 percent ammonia solutions containing 1 to $5 \mathrm{~g}$ of sodium chloride per liter may be 
used to fix the paper samples. The sodium chloride stabilized any trace of ammonia-silver complex left in the paper.

The chromatographic paper was sufficiently uniform in density that only an occasional control run would be necessary in routine testing.

A pink dye was absorbed by the paper from some films during the extraction operation. The dye was bleached out by exposing the dried paper sample for $1 \mathrm{~h}$ to a $20-\mathrm{W}$, 20-in daylight fluorescent lamp at a distance of 1 in.

The transmission density measurements reported in this paper were made with an Ansco-Sweet Densitometer, Model 11. In this paper, the concentration of residual thiosulfate is given as micrograms per square centimeter. In most of the literature, the concentration is reported in milligrams or micrograms per square inch. One microgram per square inch is equivalent to $0.155 \mu \mathrm{g} / \mathrm{cm}^{2}$.

\section{Evaluation of the Paper Extraction Method}

In evaluating the paper extraction method for the determination of residual thiosulfate in processed film, six microfilms, two motion picture films, two x-ray films, one aerial film, one print film, and one professional film were tested. The films were made by five manufacturers. The unexposed film samples were treated $4 \mathrm{~min}$ in a solution containing $90 \mathrm{~g}$ sodium sulfite, $52 \mathrm{~g}$ sodium carbonate monohydrate, and $5 \mathrm{~g}$ of potassium bromide per liter. This solution is typical of developers but without a developing agent. The developing agent was eliminated to avoid any reduction of silver in the gelatin layer. Then the film samples were fixed in an acid hardening bath and washed in chlorine-free tap water as described in a previous publication [4]. The excess water was removed from the film samples with wiping tissue. The washing time controlled the residual thiosulfate concentration in the films.

For comparison, the residual thiosulfate concentrations in the processed film samples were determined by the paper extraction method and the modified Crabtree-Ross method [5]. Samples for the two methods were taken adjacent to each other on the film. The thiosulfate concentrations ranged from 0.15 to $16 \mu \mathrm{g} / \mathrm{cm}^{3}$ of sodium thiosulfate. The two methods were in general agreement. However, the paper extraction method revealed the fact that the residual thiosulfate is not always evenly distributed in the gelatin layer, there being higher and lower values for residual thiosulfate concentration than the average value found by liquid extraction using the modified Crabtree-Ross method.

Tests were made to determine if any thiosulfate remained in the gelatin layer of the film after the first extraction. A second extraction was made with the paper extraction method immediately after the first extraction. The effective optical density of the silver sulfide was determined for two layers of the test paper and the residual thiosulfate concentration was read from the calibration curve shown in the next section. The results are tabulated in table 1. For 11 films, no thiosulfate remained where there had been a low original thiosulfate concentration and only a negligible amount remained where it had been high. Two films with a thick layer of gelatin retained about 5 percent in the low thiosulfate concentrations and
TABLE 1. Residual thiosulfate found in a second ammonia extraction of processed film.

Approximate micrograms of residual thiosulfate per centimeter originally found in the film after processing

$\begin{array}{llll}1.5 & 3 & 8 & 16\end{array}$

Micrograms of thiosulfate per square centimeter found in the second ammonia extraction

\begin{tabular}{|c|c|c|c|c|}
\hline $\begin{array}{l}\text { Six microfilms } \\
\text { Two x-ray films } \\
\text { One motion picture film } \\
\text { One aerial film } \\
\text { One print film }\end{array}$ & 0 & 0 & 0 to 0.15 & 0 to 0.7 \\
\hline $\begin{array}{l}\text { One motion picture film } \\
\text { One professional film }\end{array}$ & 0 to 0.07 & 0.15 & 0.7 & 1.5 \\
\hline
\end{tabular}

10 percent in the high. A third extraction detected no thiosulfate. The presence of thiosulfate in the gelatin layer after the first, second, or third extractions was also determined by processing the film, after extraction, by the procedure used by Crabtree, Eaton, and Muehler [1] for determining the thiosulfate in photographic papers. Using this method, one can detect $0.08 \mu \mathrm{g} / \mathrm{cm}^{2}$ of thiosulfate as silver sulfide by measuring the density of two layers of film with a Kodak 18A filter. No thiosulfate was detected in the film with low original concentrations and the amount found where concentrations had been higher correlated with that found in the second paper extraction.

The effect of a high concentration of silver in the fixing bath on the paper extraction of thiosulfate was investigated. Film was fixed in a fixing bath containing $4 \mathrm{~g}$ of silver per liter, the silver being added as silver bromide. The silver thiosulfate complex left in the film did not affect the extraction of the thiosulfate.

Tests were made to determine if any residual developing agents remained in processed film as these would form reduced silver in the test paper in the silver nitrate solution. Unexposed samples of microfilm, motion picture film, and x-ray film were normally processed and washed free of thiosulfate. The samples were treated in a bath containing $10 \mathrm{~g}$ sodium sulfite, $5 \mathrm{~g}$ boric acid, $10 \mathrm{~g}$ potassium aluminum sulfate, $10 \mathrm{ml}$ glacial acetic acid, $0.05 \mathrm{~g}$ metol, and $1 \mathrm{~g}$ hydroquinone per liter and washed $30 \mathrm{~s}$ in running tap water. The above bath contained chemical compounds found in a used fixing bath in addition to thiosulfate. When the paper extraction test was applied, there was no reduction of silver in the paper. The developing agents were rapidly removed from the films and therefore would not interfere with the paper extraction method.

Paper extraction does not remove all of the thiosulfate from areas on the film containing a large amount of image silver. One microfilm was flashed and developed to a density of 4.8 and washed $30 \mathrm{~min}$. The paper extraction method detected no thiosulfate in either the imagefree area or the image area, whereas the modified Crabtree-Ross method gave negative results for the clear area but $1.2 \mu \mathrm{g} / \mathrm{cm}^{2}$ of thiosulfate in the image area. A sample of the high-density area was treated $15 \mathrm{~min}$ in a 
5-percent potassium bromide solution, washed, and bleached with an untreated sample in a dichromate solution. The effective densities of the remaining silver sulfide measured for two layers with a Kodak 94 filter were 0.78 for the untreated sample and 0.72 for the one treated in potassium bromide solution. The silver sulfide density of 0.72 corresponds to the silver sulfide formed in the image during fixation and the difference of 0.06 density corresponds to thiosulfate absorbed on the image silver. These tests, made immediately after the film was washed, showed the absorbed thiosulfate was not removed by ammonia but was by potassium bromide. The amount of thiosulfate absorbed is very small. The above results indicate that the paper extraction method should be applied to an image-free area of the film or one not having more than 0.1 density of silver. The absorbed thiosulfate is unstable and reacts rapidly at high relative humidity with the silver in the image to form silver sulfide.

\section{Calibration Curve}

A calibration curve was prepared using the Whatman 3 MM chromatographic paper. A sample of the paper, cut 10 by $20 \mathrm{~cm}$, was treated $5 \mathrm{~min}$, with agitation, in two liters of a sodium thiosulfate solution in a photographic tray. Solutions containing 0.050, 0.065, 0.075, $0.10,0.15,0.2,0.3,0.4$, and $0.8 \mathrm{~g}$ of $\mathrm{Na}_{2} \mathrm{~S}_{2} \mathrm{O}_{3} \cdot 5 \mathrm{H}_{2} 0$ per liter were used. The paper sample was removed from the thiosulfate solution and blotted immediately between clean photographic blotters with pressure, blotted a second time between clean blotters, and allowed to dry flat on clean blotters, turning the sample over every 3 or 4 min for the first $30 \mathrm{~min}$ to obtain a more even distribution of the sodium thiosulfate in the paper.

The dried paper sample was cut into two parts, 5 by $10 \mathrm{~cm}$, and one part was treated, together with a control sample of the chromatographic paper containing no thiosulfate, $4 \mathrm{~min}$ in a $400-\mathrm{ml}$ solution containing $10 \mathrm{~g}$ of silver nitrate and $30 \mathrm{ml}$ of glacial acetic acid; fixed $3 \mathrm{~min}$ in each of two 400-ml solutions containing 3-percent ammonia and $5 \mathrm{~g}$ of sodium chloride per liter; and dried flat on blotter paper. The transmission densities of the paper sample containing silver sulfide and the paper control were measured. The density of the silver sulfide was obtained by subtracting the density of the control from that of the paper sample containing the silver sulfide. A single layer of the chromatographic paper had a density of $1.19 \pm 0.01$, two layers had $1.47 \pm 0.01$ with an occasional deviation of 0.02 . Treatment in the processing solutions did not change the density.

Four $21 / 2-\mathrm{cm}$ squares were cut from the second part of the sample mentioned above, adjacent to the first part and about $5 \mathrm{~cm}$ from the ends, and the residual thiosulfate was determined by the modified Crabtree-Ross test method [5]. In this test method $5 \mathrm{ml}$ of a 5-percent solution of mercuric chloride was added, with rapid stirring, to a vial containing $5 \mathrm{ml}$ of a 5-percent solution of potassium bromide and $100 \mu \mathrm{g}$ of gelatin. The thiosulfate reacted with the mercuric chloride to form a turbidity which was determined with a nephelometer. The thiosulfate concentration was determined from a calibration curve. Four squares were used in the test for sodium thiosulfate concentrations up to $2.5,2$ squares for 5 , and 1 square for $10 \mu \mathrm{g} / \mathrm{cm}^{2}$. The 5 -percent solutions of potassium bromide and mercuric chloride were added from burets. The samples of paper were treated $5 \mathrm{~min}$ in a $50-\mathrm{ml}$ beaker with $5 \mathrm{ml}$ of the potassium bromide solution, while agitating with a thin glass rod, and were transferred to a $10-\mathrm{ml}$ graduate. The samples were treated a second time with $5 \mathrm{ml}$ of the potassium bromide solution to remove any trace of thiosulfate remaining in the paper and the solution transferred to the same graduate. After the paper samples were removed from the beaker the volume of solution in the $10-\mathrm{ml}$ graduate was made up to $10 \mathrm{ml}$ with the 5 -percent potassium bromide solution to replace the solution absorbed by the paper and the 10-ml solution was transferred to the beaker in which the extraction was made. Four drops of a 0.1 percent gelatin solution and then $10 \mathrm{ml}$ of the mercuric chloride solution were added to the beaker with rapid stirring. Ten $\mathrm{ml}$ of the solution in the beaker were transferred to a vial and allowed to stand $30 \mathrm{~min}$ and the turbidity was determined with a Coleman No. 9 nephelometer. The vial was handled gently to avoid stirring up a few fibers of the paper that had settled to the bottom. The concentration of the sodium thiosulfate was determined from a calibrated curve for known concentrations of sodium thiosulfate in the potassium bromide-mercuric chloride solution. This value was

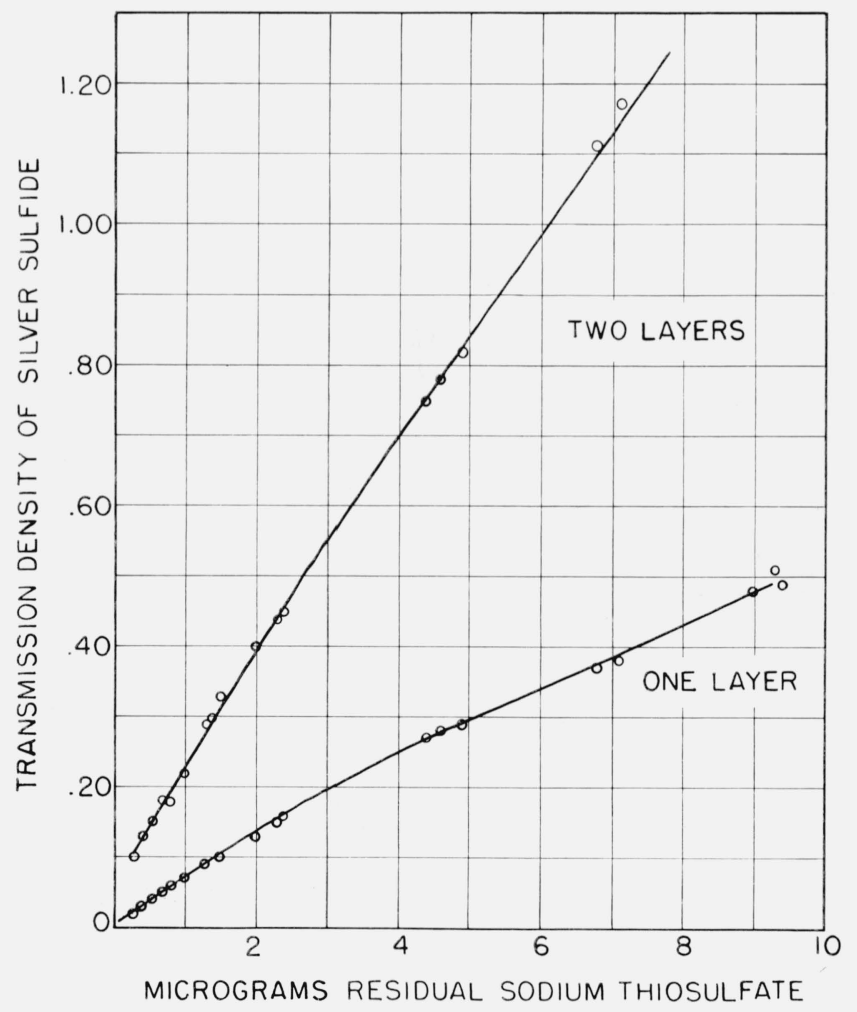

$\left(\mathrm{Na}_{2} \mathrm{~S}_{2} \mathrm{O}_{3}\right)$ PER SOUARE CENTIMETER

Figure 1. Silver sulfide calibration curve showing the transmission density of silver sulfide formed in the Whatman No. 3 MM chromatographic paper by the reaction of sodium thiosulfate with silver nitrate. 
multiplied by 2 and divided by the area in square centimeters tested to obtain the concentration of the sodium thiosulfate in micrograms per square centimeter in the paper. The transmission densities of the silver sulfide for one layer and two layers of the paper were plotted against the corresponding sodium thiosulfate concentrations to obtain the calibration curves shown in figure 1 for the Whatman No. 3 MM chromatographic paper only. Calibration curves must be prepared for each type of paper used in extracting the thiosulfate from the gelatin layer of film. The calibration procedure is very simple if a nephelometer is available for measuring turbidity.

\section{Summary}

The new paper extraction method is simple, rapid, and requires no light filters. Only a densitometer is needed as special equipment. The method is nondestructive as tests may be made on roll or sheet film which, after drying, may be returned to storage. The residual thiosulfate may be extracted in the field and the paper may be forwarded for final processing to a laboratory which has a calibration curve for the type of paper used in the extraction. The test should be made in an image-free area of the film or one not having a density greater than 0.1 . The test is quite sensitive, especially if the silver sulfide of two layers of the paper is measured. The test demonstrated that the residual thiosulfate is not always evenly distributed in the gelatin layer. Residual sodium thiosulfate as low as $0.15 \mu \mathrm{g} / \mathrm{cm}^{2}$ can be detected if the density of the silver sulfide for two layers of paper is measured. This concentration is visually discernible for one layer viewed with a control. For this reason the paper extraction test is an excellent qualitative test for the detection of residual thiosulfate in processed film. The test method will determine trithionate and tetrathionate as thiosulfate. This is advantageous since these compounds also react with the image silver to form silver sulfide.

\section{References}

[1] Crabtree, J. I., Eaton, G. T., and Muehler, L. E., A review of hypo testing methods, J. Soc. Motion Picture Engrs. 42, 34-57 (1944).

[2] Mattey, D. A., and Henn, R. W., Determination of thiosulfate and thionates in film with silver nitrate, Photo. Science and Engr. 10, No. 4, 202-208 (1966).

[3] Pope, C. I., A simplified method for determining residual thiosulfate in processed microfilm, Photo. Science and Engr. 13, No. 5, 278-279 (1969).

[4] Pope, C. I., Blemish formation in processed microfilm, J. Res. Nat. Bur. Stand. (U.S.), 72A (Phys. and Chem.), No. 3, 251-259 (1968).

[5] Pope, C. I., Determination of residual thiosulfate in processed film, J. Res. Nat. Bur. Stand. (U.S.), 67C (Eng. and Instr.), No. 3, 237-245 (1963).

(Paper 75C1-309) 